\title{
Techniques to improve the GPS precision
}

\author{
Nelson Acosta \\ Institute of Advanced Informatic Technology Research \\ University Center of the Province of Buenos Aires \\ Tandil, Argentina
}

\author{
Juan Toloza \\ Institute of Advanced Informatic Technology Research \\ University Center of the Province of Buenos Aires \\ Tandil, Argentina
}

\begin{abstract}
The accuracy of a standard market receiver GPS (Global Positioning System) is near 10-15 meters the 95\% of the times. To reach a sub-metric level of accuracy some techniques must be used [1]. This article describes some of these procedures to improve the positioning accuracy by using a low-cost GPS in a differential relative positioning way. The proposed techniques are some variations of Kalman, fuzzy logic and information selection.
\end{abstract}

Keywords- GPS accuracy; relative positioning; DGPS; precision farming GPS.

\section{INTRODUCTION}

The GPS operation principle is based on measuring ranges of distances between the receiver and the satellites [2] [3]. The GPS has architecture of three segments: spatial, control and users. The spatial includes 24 satellites over 20 thousand $\mathrm{km}$ away from the Earth, with six orbital levels and a 12-hour period. The second segment includes the Earth stations to control the satellites trajectories. Finally, the user's segment includes GPS receivers using two frequencies: L1 at 1575.42 Mhz for civil use, and L2 at 1227.60 Mhz reserved to military use [4].

The accuracy in longitude and latitude coordinates is of $10-15$ meters $95 \%$ of the readings [5]. Sometimes, it is more precise, but it depends on a variety of factors that include from the deviation or the delay of the signal when cross the atmosphere, the bouncing of the signal in buildings or its concealment due to the presence of trees [6], low accuracy of clocks and noise in the receiver. In altitude the accuracy is reduced to $50 \%$ regarding the obtained in terms of longitude and latitude (15-23 meters 95\%) [7].

Systems that enhance positional accuracy are: the DGPS (Differential GPS), AGPS (Assisted GPS), RTK (Real-Time Kinematic), e-Dif (extended Differential), amongst others.

The DGPS corrections service has two hard restrictions: it must be afforded and the receiver must be close to a DGPS station (less than $1000 \mathrm{~km}$ ). The achieved accuracy can be of a few meters $[7,8,9,10]$. The correction signal cannot be received if it is a mountainous zone.

In the case of AGPS, it is necessary to have mobile devices with active data connection or cell phone like GPRS, Ethernet or $\mathrm{WiFi}$ [11]. It is used in the cases where there is a weak signal due to a surrounding of buildings or trees; this implies having a not much precise position. Standard GPS receivers, in order to triangulate and position, need a certain time of cold start [12][13]. a) For use the RTK system, it is paid for the service and, besides, it is very expensive to acquire the infrastructure. This is a technique used in topography, marine navigation and in agricultural automatic guidance in the use of measurements of signals carrying navigators with GPS, GLONASS (Globalnaya Navigatsionnaya Sputnikovaya Sistema) and/or Galileo's signals, where only one reference station provides correction in real time, obtaining a sub-metric accuracy [14].

b) The last case, e-Dif system, is autonomous and it process files with RINEX (Receiver Independent Exchange) format, which was created to unify data of different receivers manufacturers [15]. It generates autonomous corrections regarding a coordinate of arbitrary reference and it extrapolates them in time [16]. It is a very consistent relative positioning and its accuracy is of about 1 meter. The system's objective is to study waste from the initializing process to isolate the most important systematic errors that introduce the corresponding equations to each satellite. It is applicable for a reduced time of 40 minutes approximately; since later the systematic error changes, in this case a new error must be calculated again. In regions where differential corrections aren't available and it is paid for the service, like in South American, African and Australian, this system become more interesting.

c) Besides, there are systems that increase accuracy to sub-metric levels. Those based on satellite SBAS (SatelliteBased Augmentation System), based on ground GBAS (Ground-Based Augmentation System) and based on aircraft ABAS (Aircraft-Based Augmentation System). Most of these implementations are used in different applications and some of them are available for users without special permissions. Even then, costs are high due to the need of certain devices with special characteristics or some infrastructure in agreement with the accuracy level desired.

Errors produced by the GPS system affect in the same way the receivers located near each other in a limited radius. This implies that errors are strongly correlated among near receivers. Thus, if the error produced in one receiver is known, it can be spread towards the rest in order to make them correct their position. This principle is only applicable to receivers that are exactly the same, the same methodology of [17]; since, if different, their specifications change so the signal processed by one individual is not the same to that processed by another one. 
All GPS differential methods use the same concept [18]. DGPS requires a base station with a GPS receiver in a precise known position. The base compares its known position with that calculated by the satellite signal. The estimated difference in the base is applicable then to the mobile GPS receiver as a differential correction with the premise that any two receivers relatively near experiment similar errors [5].

In this article it is emphasized in the behavior of GPS errors in time, after the techniques developed here to determine magnitude and direction of error are applied. In Section 2 techniques to calculate errors are analyzed. In Section 3 algorithms used are described and finally the error behavior is presented in figures. In Section 4, the last one, conclusions and future work are presented.

\section{TECHNIQUES FOR ERROR ANALYSIS}

The experiment carried out is based on the principle of the adopted methodology by the DGPS but with a low cost standard GPS receiver. In order to get measurements, three Garmin 18X USB GPS receivers are used connected to two notebooks. The base station is composed of a notebook and two of the three GPS receivers; the mobile for the other notebook and the remaining GPS receiver. The link between the base station and the mobile one is a point to point wireless connection.

In this context, in the base system, measurements from the GPS receivers are obtained and after a certain period of time, which is necessary for the system stabilization, two positions are estimated. The positions' estimation are carried out with a Kalman filter, since an estimation problem with so many noisy redundant data is a natural application for the Kalman filter; this allows using some of the redundant information to remove the effects from the error sources. The Kalman filter is used to eliminate the white Gaussian noise [19].

Receivers are placed at a known distance between themselves (relative positioning) [20]. At the end of this stage, a cloud of points from standard GPS system is obtained with the positions delivered by the receivers and with those values a position is estimated for each receiver. The estimated point from GPS 1 is selected as anchor point of the whole experiment. From this, all necessary calculations are carried out with the objective of finding the GPS system error.

With both estimated positions, it proceeds to calculate the distance between them. If the estimated distance is different from the actual one (more/less a threshold) it is detected that there is a positioning error. Besides, a circumference with a radius equal to the actual distance measured with a tape measure from GPS 1 to GPS 2 with center in the estimated point for the GPS 1 is drawn. A circumference is chosen, as GPS 2 can be at that distance but in any point of the circumference. This is the working principle that the GPS system uses to get the receiver's position.

After calculating the distance of the estimated points and contrasting it to the actual one, a positioning error is deduced. Once it is known that there is an error, it must compute its magnitude and direction. On the one hand, the two estimated points are learnt with which the straight line is drawn and which bonds them. Equation 1 belongs to the straight line that crosses these two points.

$$
\frac{(y-y 1)}{(y 2-y 1)}=\frac{(x-x 1)}{(x 2-x 1)}
$$

where the $x$ represents the component of Longitude and the $y$ that of Latitude.

On the other hand, it is known that the GPS 2 is in some point of the circumference with center in the GPS 1 and of radius the distance that was defined at the moment of positioning the two receivers. Equation 2 belongs to the circumference with center in $(x 1, y l)$ of radius $\mathrm{r}$.

$$
(x-x 1)^{2}+(y-y 1)^{2}=r^{2}
$$

Knowing about the equations that define the straight line crossing both estimated points and the radius circumference equation equal to the GPS 1-GPS 2 distance with center in GPS 1, it is proceeded to approximate, by means of the intersection of the straight line and the circumference.

This intersection is presented as a polynomial of second degree. It is mathematically solved and $a, b$ and $c$ coefficients are obtained (equations 4, 5 and 6). Equation 3 only presents an auxiliary estimate in order not to repeat it in the other operations and to increase legibility in the rest of the equations. With these coefficients cleared by means of Bascara (equation 7) the roots are found (two because of being of second degree) from the polynomial. From the two roots found, one is chosen and the intersection points are calculated.

$$
\begin{gathered}
\text { divisor }=y_{2}^{2}-2 y_{2} y_{1}+y_{1}^{2} \\
a=1+\frac{\left(x_{2}^{2}-2 x_{2} x_{1}+x_{1}^{2}\right)}{\text { divisor }} \\
b=-2 y_{1}+\frac{\left(-2 x_{2}^{2} y_{1}+4 x_{2} x_{1} y_{1}-2 x_{1}^{2} y_{1}\right)}{\text { divisor }} \\
c=y_{1}^{2}-r^{2}+\frac{\left(x_{2}^{2} y_{1}^{2}-2 x_{2} x_{1} y_{1}^{2}+x_{1}^{2} y_{1}^{2}\right)}{\text { divisor }} \\
x=\frac{-b \pm \sqrt{\left(b^{2}-4 a c\right)}}{2 a}
\end{gathered}
$$

After having obtained the two roots, only one is taken into account. The nearest to the estimated point of GPS 2 in some of its components is chosen, in this case in Latitude, since the other one is meaningless due to being too far away (on the other side of the circumference).

$$
\text { result }_{\text {latitude }}=\text { rootCloser }\left(y_{2}, \text { roots.root }_{1}, \text { roots.root }_{2}\right)
$$

Finally, the other component (Longitude) is obtained depending on the Latitude found in the previous point as seen in equation 8. 


$$
\text { result }_{\text {longitude }}=x_{1}+\left(\left(x_{2}-x_{1}\right) * \frac{\left(\text { result }_{\text {latitude }}-y_{1}\right)}{y_{2}-y_{1}}\right)
$$

Once the error magnitude is known, is necessary to get the direction in order to have the final correction vector. There are nine possible cases, that are the combination of two variables (latitude and longitude) and three values that are: greater, lesser and equal. In Table I these cases are presented.

TABLE I. DETERMINATION OF ERROR DIRECTION.

\begin{tabular}{|c|c|}
\hline \multicolumn{2}{|c|}{ Estimated } \\
\hline Latitude & Longitude \\
\hline \hline Greater & Greater \\
\hline Greater & Lesser \\
\hline Greater & Equal \\
\hline Lesser & Greater \\
\hline Lesser & Lesser \\
\hline Lesser & Equal \\
\hline Equal & Greater \\
\hline Equal & Lesser \\
\hline Equal & Equal \\
\hline \hline Latitude & Longitude \\
\hline \multicolumn{2}{|c|}{ Actual } \\
\hline \multicolumn{2}{|c}{}
\end{tabular}

The way to read the table is top-down. Estimated latitude greater than actual latitude and an equal longitude to actual is presented in the third row. In the case where actual and estimated components are equal, like in ninth row of the Table $\mathrm{I}$, there is no correction.

Therefore, in order to get the error direction it is proceeded to verify which side of the corrected point the estimated point is. If the straight line that joins both estimated points is horizontal or vertical, some of the components are null, in the horizontal case, Latitude is eliminated and in the vertical the Longitude.

\section{ALGORITHMS AND USED TECHNIQUES}

For the analysis of data, combinations of different techniques and algorithms are used in order to find a better result. In a first processing stage, applied mathematics covers:

- Static Kalman: is a set of mathematical equations that provide an efficient recursive solution of the method of least squares. This solution allows calculating a linear, unbiased and optimum estimator of the state of a process in each moment of time $(t)$ with base on the available information at the moment $t-1$, and update, with the available information at the moment $t$, the estimator value.

- Dynamic Kalman: is the system in which the value of variable $x$ to be estimated has a value that changes throughout the time $\left(x_{i+1} \neq x_{i}\right)$, but these states have some known relationship with the instant $i$ and $i+1$.
For example, if an object position is measured, it can be predicted that the position will be:

$$
x_{i+1}=x_{i}+\Delta t^{*} v_{i}
$$

where $\Delta t$ is the passed time and $v_{i}$ the speed at instant $i$. Position can be obtained by a GPS, for instance, and speed with an additional measurement element such as an accelerometer.

- Kalman with adjustment of error standard deviation: the deviation is modified and checked in order to see which adjust better. This measure is calculated as the square root of variance, which is at the same time the sum of the squares of each error (Table II) as shown in equation 9. It is worth mentioning that from Table II the only error that is not taken into account is that of signal $\mathrm{P}(\mathrm{Y})$ arrival; since work is carried out without the precision code.

$$
\sigma_{R}=\sqrt{3^{2}+5^{2}+2.5^{2}+2^{2}+1^{2}+0.5^{2}} m=6,7 m
$$

TABLE II. GPS ERROR SOURCE.

\begin{tabular}{|l|l|}
\hline \multicolumn{1}{|c|}{ Source } & \multicolumn{1}{c|}{$\begin{array}{c}\text { Effect } \\
\text { (Meters) }\end{array}$} \\
\hline Arrival of signal C/A & \pm 3 \\
\hline Arrival of signal P(Y) & \pm 0.3 \\
\hline Ionosphere & \pm 5 \\
\hline Ephemeris & \pm 2.5 \\
\hline Satellite clock error & \pm 2 \\
\hline Multipath & \pm 1 \\
\hline Troposphere & \pm 0.5 \\
\hline Numerical errors & \pm 1 \\
\hline
\end{tabular}

Now, error standard deviation $(\sigma)_{c}$ in the receiver's position is estimated, but having into account additionally the PDOP (Position Dilution of Precision) and the numerical error; therefore, the PDOP is added to the calculated deviation from typical errors, since for each measurement taken, this varies according to the instant geometry of satellites. The result of standard deviation used for the Kalman filter is equation 10 .

$$
\sigma_{r c}=\sqrt{P D O P^{2} * \sigma_{R}^{2}+\sigma_{\text {num }}^{2}}=\sqrt{P D O P^{2} * 6.7^{2}+1^{2}} m
$$

The fact of applying Kalman with adjustment of standard deviation, since it fluctuates for each piece of information coming from the receivers in each moment as geometry of satellites varies.

- Points average: one of the media limitations is that it is affected by extreme values; very high values tent to increase it while very low values tend to reduce it; this implies that it may stop being representative of the population. It is analyzed but not implemented in the solution. This solution was used in [21].

- Fuzzy logic: to determine the position error degree. Rules that determine the position error are related to analyzing some parameters like: PDOP, SNR (Signal-to-Noise Ratio) and difference of tracked 
satellites. The fuzzy system output weights the Kalman filter gain, providing more weight to more precise positions and the other way round.

- Filters allow discarding measurements with much noise or error that influence over the final result of an estimation of a position. Thus, measurements having many errors do not slant the final estimation towards a position far away from the actual one. The application of these filters can be made as measurements are not very far away in time and it is supposed that the Vehicle in which the mobile receiver is placed does not move at high speed; this implies that values do not change radically. High/low step filters are used in an analogical way to the electronic filter.
After apply the current techniques, in Figs. 1, 2 and 3 it is observed the original and estimated errors. The absolute errors are calculated by means of relative positioning determined by sets of two receivers. The distance between the pairs of receivers is known at starting the experiment. In Fig. 1, the estimated error tends to zero. By the other hand, in Fig. 2, estimated error fluctuates around the average of the original error. Finally, in Fig. 3, the estimated error is zero in a moment, but after a period, begins to oscillate. With this set of graphics, it is observed an oscillation in the standard system that doesn't allows having a known error to correct a position. When the presented techniques are applied it is possible to obtain an smoothing error, a value that fluctuates less in time, which allows to correct positions in other receivers in order to improve the accuracy.

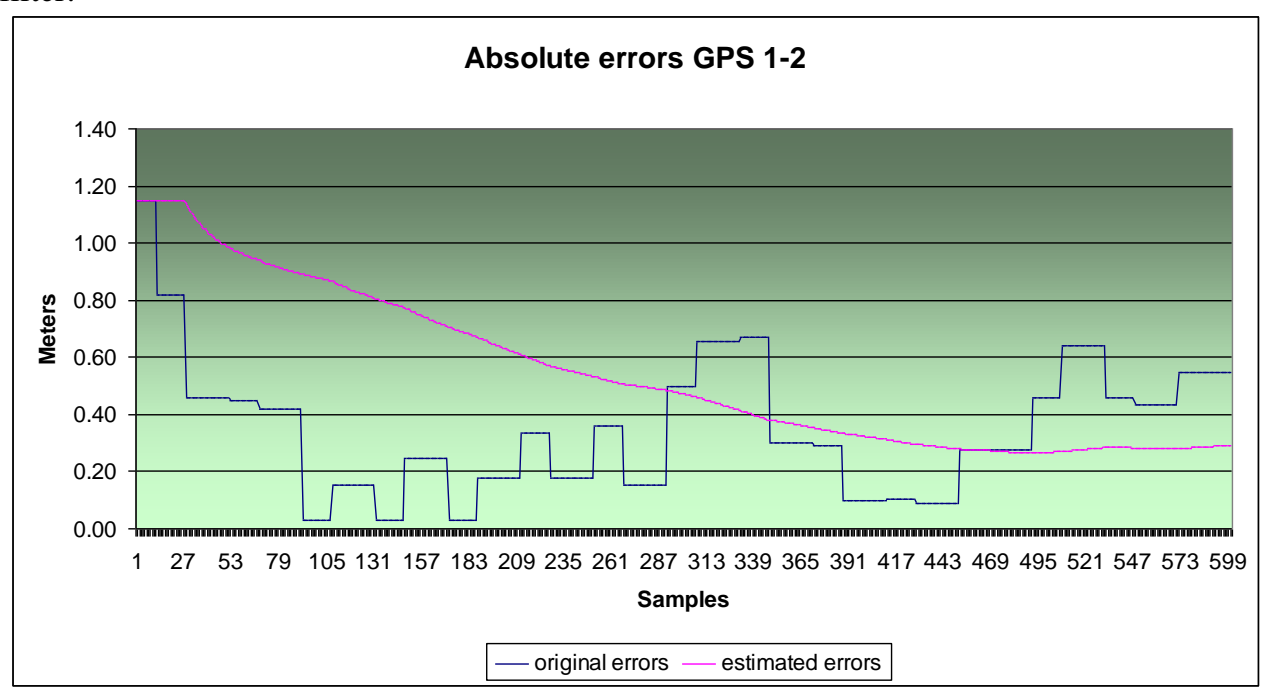

Figure 1. Behavior in time of estimated and original errors for GPS 1 regarding to GPS 2.

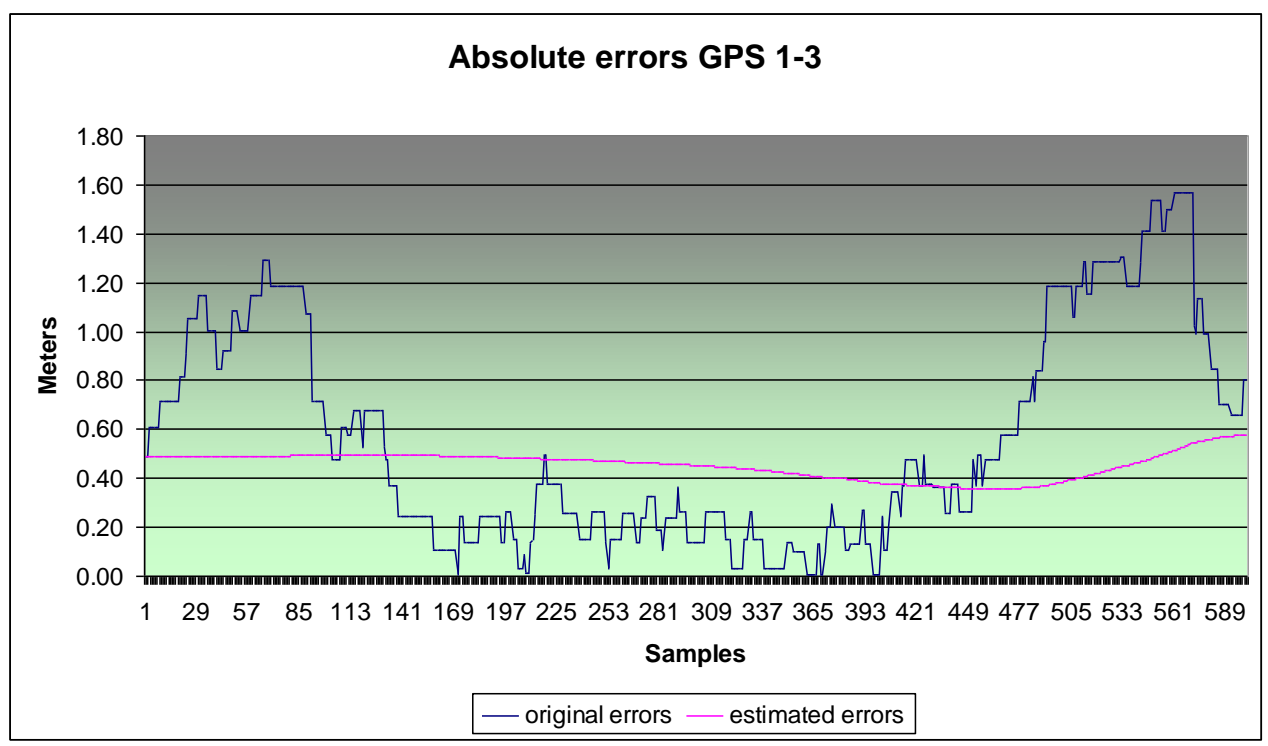

Figure 2. Behavior in time of estimated and original errors for GPS 1 regarding to GPS 3. 


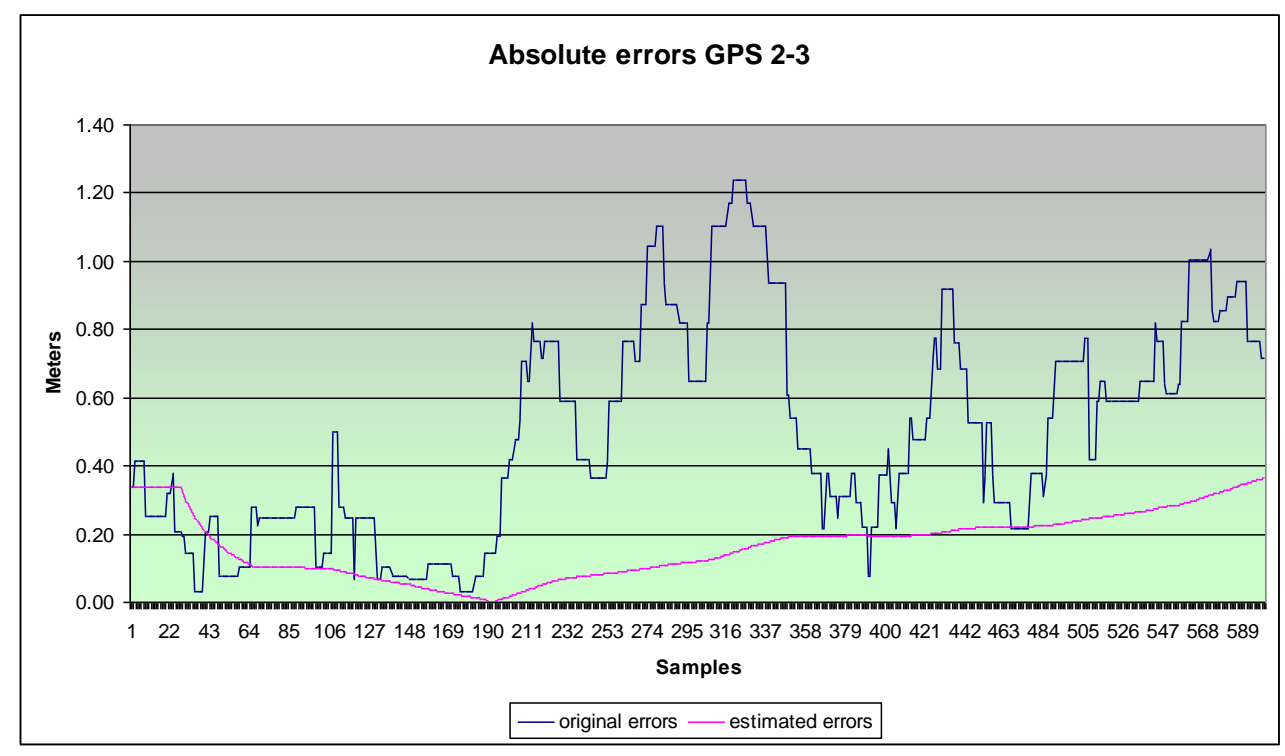

Figure 3. Behavior in time of estimated and original errors for GPS 2 regarding to GPS 3.

Since the tool that implements these techniques is thought to operate in different places of heterogeneous characteristics, relations and configurations are used in order to be able to customize the use according to needs. Relations and configurations used are the following:

- Rate Degree/Meters in Latitude: given the asymmetry, in different places on Earth, the distance that a Latitude degree measures varies.

- Rate Degree/Meters in Longitude: ditto to Latitude.

- Cold start time: a start time is considered so the system can be stabilized. In this time, samples of the device are taken and, only at its end, estimation is carried out. The objective is to reduce or soften systematic and random errors of the GPS system.

- Receivers' distance threshold: it can be determined, in an accurate way, the whole interval of the distance measurement between the base devices. As positioning is relative and its distance is known, it can be added a \pm value, since there exists a possibility that an element of distance measurement be not accurate enough. Besides, it reduces the computational load because of not having to process data if distance is within the allowed threshold.

\section{CONCLUSION AND FUTURE WORKS}

The techniques developed allow obtaining the magnitude and direction of error provoked by the GPS system as presented in [21]. This correction is used by another receiver to correct its own position and thus increase the positional accuracy with the aim of measuring the most precise distances.

The experiments carried out with different sets of data provide positions that are used to measure distances and error fluctuates in \pm 1 meter the $95 \%$ of measurements and in some cases in \pm 0.2 meters.
The principle is based in mathematical, geometrical functions and filters. With the techniques presented in this article, it is possible to obtain a smoothing error and a value constant in time, which allows correcting positions in other receivers in order to improve their accuracy.

As future work, the aim is to increase the accuracy until reaching a maximum error of positioning of \pm 0.1 meters. This increase can include the use of another additional signal. Besides, further measurements will be carried out in order to analyze data and to deduce its behavior. It is intended to extend the use in faster vehicles in order to widen the application field of the DGPS system introduced here.

\section{ACKNOWLEDGMENT}

Agencia Nacional de Promoción Científica y Tecnológica for supporting since 2009.

\section{REFERENCES}

[1] M. S. Grewal, L. R. Weill and A. P. Andrews, Global Positioning Systems, Inertial Navigation, and Integration. $2^{\text {nd }}$ Edition, Wiley, 2007.

[2] G. Xu, GPS: Theory, Algorithms and Applications. 2 ${ }^{\text {nd }}$ Edition, Springer-Verlag Berlin Heidelberg, 2007.

[3] P. Misra and P. Enge, Global Positioning System: Signals, Measurements, and Performance. New York, Ganhga-Jamuna Press, 2010.

[4] T. Feldmann, A. Bauch, D. Piester, H. Esteban, J. Palacio, F. J. Galindo, T. Gotoh, H. Maeno, U. Weinbach and S. Schon, "GPS carrier phase and precise point positioning time scale comparisons using different software packages," Frequency Control Symposium, 2009 Joint with the $22^{\text {nd }}$ European Frequency and Time Forum, IEEE, pp. $120-125,2009$.

[5] P. A. Zandbergen and L. L. Arnold, "Positional accuracy of the wide area augmentation system in consumer-grade GPS units," Computers and Geosciences Volume 37 Issue 7, Elsevier, pp. 883-892, 2011.

[6] C. Ordónez Galán, J. R. Rodríguez-Pérez, J. Martínez Torres and P. J. García Nieto, "Analysis of the influence of forest environments on the accuracy of GPS measurements by using genetic algorithms," Mathematical and Computer Modelling Volume 54 Issue 7-8, Elsevier, pp. 1829-1834, 2011. 
[7] S. Featherstone, Outdoor Guide to Using Your GPS. Creative Publishing International, Inc., 2004.

[8] G. Satheesh, Global Positioning Systems: Principles and Applications. Mc-Graw Hill, 2005.

[9] S. D. Ilčev, Global Mobile Satellite Communications for Maritime, Land and Aeronautical Applications. Springer, 2005.

[10] M. Ghavami, L. B. Michael and R. Kohno, Ultra Wideband Signals and Systems in Communication Engineering. $2^{\text {nd }}$ Edition, John Wiley and Sons, Ltd., 2007.

[11] C. Ho, "An effective approach in improving A-GPS accuracy to enhance hybrid positioning computation," $17^{\text {th }}$ International Conference on Embedded and Real-Time Computing Systems and Applications (RTCSA), Toyama, Japan, IEEE, pp. 126-130., 2011.

[12] J. $\mathrm{Li}$ and $\mathrm{M}$. Wu, "A positioning algorithm of AGPS," International Conference on Signal Processing Systems, Singapore, IEEE, pp. 385388, 2009.

[13] F. Van Diggelen, A-GPS, Assisted GPS, GNSS, and SBAS. Artech House, 2009.

[14] D. Dardari, E. Falletti and M. Luise, Sattellite and Terrestrial Radio Positioning Techniques: A Signal Processing Perspective. $1^{\text {st }}$ Edition, Elsevier, 2012.

[15] N. H. M. Hanif, M. A. Haron, M. H. Jusoh, S . A. M. Al Junid, M. F. M. Idros, F. N. Osman and Z. Othman, "Implementation of real-time kinematic data to determine the ionospheric total electron content," $3^{\text {rd }}$ International Conference on Intelligent Systems Modelling and Simulation (ISMS), Kota Kinabalu, Malaysia, IEEE, pp. 238-243, 2012.

[16] GPS World (serial online), "CSI wireless differential software patented," Volume 13 Issue 8, EDS Foundation Index, Ipswich, MA. pp. 48, 2002. Accessed June 18, 2012.

[17] M. G. Wing and J. Frank, "Vertical measurement accuracy and reliability of mapping-grade GPS receivers," Computers and Electronics in Agriculture Volume 78 Issue 2, Elsevier, pp. 188-194, 2011.

[18] V. Di Lecce, A. Amato and V. Piuri, "Neural technologies for increasing the GPS position accuracy," International Conference on Computational Intelligence for Measurement Systems And Applications (CIMSA), Istanbul, Turkey, IEEE, pp. 4-8, 2008.
[19] H. Eom and M. Lee, "Position error correction for DGPS based localization using LSM and Kalman filter," International Conference on Control, Automation and Systems (ICCAS), Gyeonggi-do, Korea, IEEE, pp. 1576-1579, 2010.

[20] Y. He, H. Yu and H. Fang, "Study on improving GPS measurement accuracy," Instrumentation and Measurement Technology Conference (IMTC), Otawa, Canada, IEEE, pp. 1476-1479, 2005.

[21] J. Toloza, N. Acosta and A. De Giusti, "An approach to determine the magnitude and direction error in GPS system," Asian Journal of Computer Science and Informatin Technology, in press, 2012.

\section{AUTHORS PROFILE}

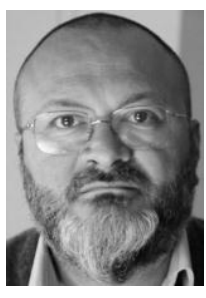

Ph.D. Héctor Nelson Acosta was graduated at the Universidad Nacional del Centro de la Provincia de Buenos Aires (Argentina), and got the PhD degree at the Autonomous University of Madrid (Madrid, Spain). $\mathrm{He}$ is the director of the Instituto de Investigación en Tecnología Informática Avanzada (INTIA) at the Universidad Nacional del Centro de la Provincia de Buenos Aires since the 1998. He is working as a proffesor since 1993 at the Computer and Systems Department, at the Universidad Nacional del Centro de la Provincia de Buenos Aires, Tandil (Argentina). He has been a visiting proffesor of several Universities in Argentine. He has suppervised 6 $\mathrm{Ph} . D$. students in Computer Sciences and $2 \mathrm{MSc}$ in Informatics. His research insterest includes real-time systems, signal procesing, pattern recognition, custom architectures, custom processors, robotic navigation and accuracy positioning systems.

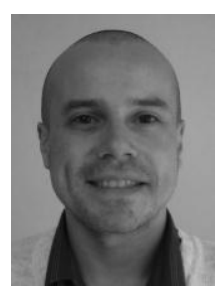

Eng. Juan Manuel Toloza was graduated in march 2009 at the Universidad Nacional del Centro de la Provincia de Buenos Aires (Argentina). He is in the last year of Ph. D. (Computer Science) at the Universidad Nacional de La Plata (Argentina). He have a scholarship of the Agencia Nacional de Promoción Científica y Tecnológica since 2009. He works in the Instituto de Investigación en Tecnología Informática Avanzada at the Universidad Nacional del Centro de la Provincia de Buenos Aires. His research insterest includes signal procesing, data analysis from diferents sensors, robotics and accuracy GPS. He is teacher assistant in Computer`s Architecture and Digital Techniques. 